\title{
Stoelting's Handbook of Pharmacology \& Physiology in Anesthetic Practice - 3rd Edition
}

\author{
Robert K. Stoelting, Pamela Flood, James P. Rathmell, Steven Shafer. Wolters Kluwer \\ Health, 2015, 808 pages. ISBN-13: 978-1605475493
}

\author{
Jacques Somma, MD
}

Received: 8 October 2015/Accepted: 14 October 2015/Published online: 19 October 2015

(C) Canadian Anesthesiologists' Society 2015

This 808-page handbook is a condensed version of the fifth edition of the textbook, Stoelting's Pharmacology \& Physiology in Anesthetic Practice, and follows an identical structure with the same chapter titles and headings as in the main textbook. The handbook is intended to "serve as a companion" and to provide, as stated in Dr. Stoelting's preface, "a rapid and accurate source of information" relevant to the pharmacology and physiology that clinicians will face in their anesthesiology practice.

The physical size of the handbook is ideal to put in the pocket of a typical white lab coat. While the book has a softcover, both the bookbinding and the pages are of excellent quality. In addition, the page corners are rounded, which helps to protect the handbook. The font size and the quality of the color figures are excellent, and each of these attributes contributes to the comfortable handling and reading of this book.

The handbook consists of 47 chapters grouped into nine parts, with each part comprising as few as two chapters to as many as eleven. Part I is a very brief (two chapters) section on the basic principles of physiology and pharmacology. Parts II to VII are divided into physiologic systems (neurologic, circulatory, pulmonary, blood and hemostasis, gastrointestinal and metabolism, endocrine), with nephrology included with the circulatory system. The last two parts of the handbook, Parts VIII and IX, are dedicated to miscellaneous topics (e.g., antimicrobial agents, chemotherapeutic drugs, drugs for

\footnotetext{
J. Somma, MD ( $\square)$

Institut universitaire de cardiologie et de pneumologie de Québec (IUCPQ), Université Laval, Québec, QC, Canada

e-mail:sommaduke@yahoo.com
}

psychopharmacologic therapy) and special populations (e.g., newborns, maternal and fetal physiology and pharmacology, the elderly, and resuscitation).

Parts II to VII, dedicated to physiologic systems, represent $80 \%$ of the book and follow a similar structure. The first chapter typically describes the physiology of the system and the following chapters are organized by drug classification. The two most sizeable parts are Parts II and III on the neurologic and circulatory systems, respectively. The "Neurologic System" consists of eleven chapters. Its first chapter, "Neurophysiology", covers the full range of neurologic aspects, including nerve and neurotransmitters physiology; brain, brainstem, and spinal cord anatomy; the sympathetic and parasympathetic nervous system; the chemoreceptor trigger zone; electroencephalogram and evoked potentials; and thermoregulation. The related chapters that follow include inhaled anesthetics, intravenous sedatives and hypnotics, pain physiology, opioids, nonopioid analgesics, local anesthetics, neuromuscular physiology and blocking agents, and antiepileptic drugs.

The systemic approach is ideal for the anesthesia trainee as there is a logical progression from the physiology of a system to the pharmacology of the drugs being used on that system. While not perfect, this handbook is relatively comprehensive for such a compact manual. Nevertheless, by condensing the text for brevity, some explanations of concepts and figures have been omitted, occasionally at the expense of clarity. In some cases, I found it necessary to read the main textbook to understand some of the figures or concepts presented in the handbook. In other cases, entire sections of the textbook were completely omitted. While most of these omissions are justified, one or two sentences to describe some of these topics could have been included 
and would have contributed valuable information. For example, in the current textbook, there is an interesting section on cardiac protection and ischemic preconditioning that describes the potential cardiac protective effect of volatile anesthetics. This was omitted in the handbook, although an entire page is used for a figure from a 1978 manuscript describing the circulatory effect of enflurane. Another notable omission is a discussion on the physiology and treatment of malignant hyperthermia. It was somewhat disappointing to see certain typos, including some errors in tables that were incorrectly reproduced from the textbook.

Finally, as for all previous editions of the textbook, there are two indexes, a drug index and a subject index, which should help the reader search for information more efficiently. I did find, however, that the usefulness of these indexes is somewhat hindered by the lack of completeness and other typos. For example, the information regarding preoperative preparation of patients with allergic histories is found in the chapter "Gastrointestinal Mobility Drugs", and a paragraph on treating anaphylactic reaction is found in the chapter "Neuromuscular Blocking Drugs". Searching in both indexes for anaphylaxis, allergy, or even epinephrine was fruitless. This information could be found only by reading the handbook from cover to cover. Another such example is not being able to find an especially useful table that describes the management of local anesthetic toxicity strictly by searching only the indexes.

These limitations aside, this handbook is well organized, beautifully illustrated, pleasant to read, and packed with useful and concise information. It can easily be kept on hand and read during short periods of free time. This handbook would be useful to residents and anesthesiologists who want to refresh their memory on the physiology and the pharmacology of drugs used in anesthesiology, though I would recommend the handbook as a supplement to the main textbook since some sections are more clearly understood from reading the textbook.

Conflicts of interest None declared. 